\title{
Algorithms for balancing demand-side load and micro-generation in Islanded Operation
}

\author{
Albert Molderink, Vincent Bakker, Johann L. Hurink, Gerard J.M. Smit \\ Department of Computer Science, Mathematics and Electrical Engineering, University of Twente \\ P.O. Box 217, 7500 AE, \\ Enschede, The Netherlands \\ a.molderink@utwente.nl
}

\begin{abstract}
Micro-generators are devices installed in houses producing electricity at kilowatt level. These appliances can increase energy efficiency significantly, especially when their runtime is optimized. During power outages microgenerators can supply critical systems and decrease discomfort.

In this paper a model of the domestic electricity infrastructure of a house is derived and first versions of algorithms for load/generation balancing during a power cut are developed. In this context a microCHP device, producing heat and electricity at the same time with a high effciency, is used as micro-generator.

The model and the algorithms are incorporated in a simulator, which is used to study the effect of the algorithms for load/generation balancing. The results show that with some extra hardware all appliances in a house can be supplied, however not always at the preferred time.
\end{abstract}

\section{Introduction}

It is foreseen that in the coming years the electricity generation will change thoroughly. Nowadays most electricity is generated centrally in large power plants and distributed to the consumers via the grid. The efficiency of central generation is at most 55\% due to inefficient generation [3] (transport losses not taken into account). The low efficiency is mainly caused by dumping heat — produced as byproduct - and high fluctuations in demand $[3,6]$.

The growing awareness of the greenhouse gas effect and increasing energy prices require efficiency improvements of electricity production, distribution and consumption. Therefore a shift towards decentralized electricity production is expected, especially by micro-generators [3]. These devices generate electricity at kilowatt level in or nearby houses resulting in less transport losses and better optimization potential for matching demand and supply. Furthermore micro-generators are more energy efficient than conventional power plants and some are based on renewable energy sources $[8,3]$.

A micro-generator installed within a house is also advantageous during a power cut. In such a situation the house is disconnected from the grid and the micro-generator can be started. We call this Islanded House. As the microgenerators have a limited generation capacity, the most important appliances such as heating and lighting should be supplied. The rest of the generated electricity can be used to supply other appliances to decrease discomfort.

Today, a lot of different micro-generators are available, e.g. solar cells, micro-windgenerators, micro-gasturbines and microCHP devices. The optimization potential depends on the type of micro-generator, in particular on the scheduling freedom. When and for how long a micro-gasturbine is running can be controlled in detail, whereas solar cells and micro-windgenerators have no freedom at all.

The coming years microCHP devices will replace the conventional gas-fired high-efficiency boilers producing next to heat also electricity [8]. The advantage of this type of micro-generator is that the produced heat is used for central heating and hot-water taps and the electricity is used in house or exported to the grid. This results in an efficiency of up to $95 \%$, although it still consumes conventional fuel. The appliance is heat driven, i.e. in the microCHP concept electricity is seen as byproduct (electricity can be imported and exported, while heat cannot). The ratio between heat and electricity is fixed, for current available microCHP devices the heat-electricity ratio is around 8:1.

Just replacing a conventional boiler with a microCHP device already results in a significant reduction of energy usage [5]. But a microCHP device does have even more potential when the runtime is controlled. If next to a microCHP device also a hot-water tank is installed, the heat 
and electricity production are decoupled, since heat can be produced before it is used. The total runtime of the microCHP device is defined by the total heat consumption, subject to the enlarged constraint that the heat must be produced before or at the moment it is consumed. In this way a microCHP has some limited amount of scheduling freedom. For our research we focus on a microCHP device as micro-generation, but the algorithms are designed such that they are also applicable to other micro-generators.

In this paper a model of the domestic electricity infrastructure and the electricity streams is presented. Furthermore, first versions of algorithms to balance the domestic load and micro-generation in Islanded operation are presented. Since the defined model consists of the total electricity infrastructure of a house also the non-Islanded situation (normal operation) is covered.

In the next section, first a model of the domestic electricity infrastructure is defined and criteria and objectives for the control algorithm are derived. Next, multiple algorithms are given, which decide when to switch the microCHP device on or off and which appliances to supply with the produced electricity. A simulator, based on the model, is built to study the effects and results of different algorithms. The last section gives the results of first simulations.

\section{Approach}

In this section a model of the domestic electricity infrastructure is presented. We start with introducing the required additional hardware. Next, in Section 2.2, the basic model is presented. In the last subsections subsequently the optimization decisions, the objective and the algorithms are discussed.

\subsection{Domestic Infrastructure}

To become (partly) self supporting during power cuts some changes/additions to the domestic infrastructure are necessary. We assume that a microCHP device only has a few generation modes (usually on/off) which gives limitations on the generation level. Furthermore, we assume that appliances can only be switched on/off entirely. This gives limitations on the amount of electricity shaved. Therefore, generation and consumption can hardly ever be exactly matched. An electricity buffer is required to bridge the gap between generation and consumption [7].

The proposed changes are an Uninterrupted Power Supply (UPS) functionality and additional hardware in every power outlet (see Figure 1). The outlets are extended with measuring, switching and communication hardware. First versions of these technologies are already commercially available [1].

The UPS has two functions. First, when in case of a power failure the house is disconnected from the grid it

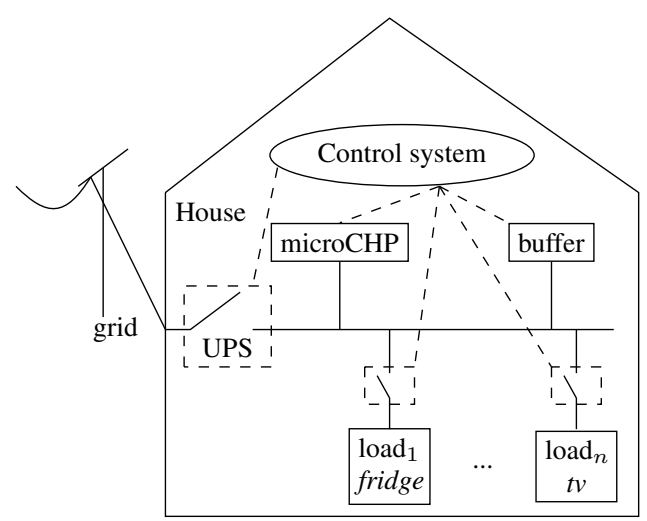

Figure 1. Proposed infrastructure

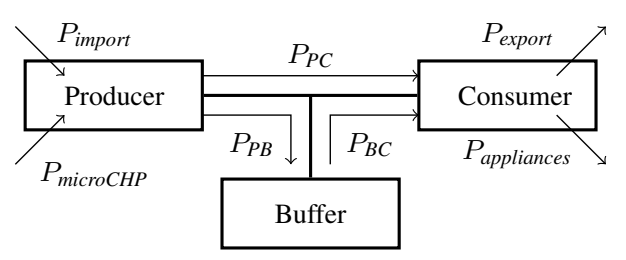

Figure 2. Model of electricity streams

takes care of the supply until the microCHP device has been started. Second, it provides an electricity buffer capacity (a UPS contains a battery). Electricity surplus is stored in the battery, generation shortage is supplied from the battery. When the battery has enough capacity, the microCHP device may be able to stop temporarily because on average the generation capacity of a microCHP device during a day is two times the daily demand in the Netherlands [5].

\section{$2.2 \quad$ Model}

The model of the house comprises the total domestic electrical infrastructure, including the connection to the grid. It consists of three main blocks: production, consumption and buffering (see Figure 2). The production comprises micro-generators and import from the grid. Consumption includes supplied appliances and export to the grid. In the figure the directions of the electricity streams are shown: production supplies consumption and fills the buffer, consumption is supplied by the production and the buffer.

Within the model, we discretisize the planning horizon, resulting in a set $\mathrm{T}=\left\{\mathrm{t}_{0}, \ldots, \mathrm{t}_{N_{T}}\right\}$ of relevant time intervals, where $t_{i+1}$ follows directly after $t_{i}$. The number of intervals depends on the length of the planning horizon and the length of the intervals. 


\section{Appliances}

We assume that the total electricity consumption results from a set of appliances. The request of an appliance is specified by an earliest (and preferable) start time, a runtime and a consumption profile. The consumption profile is a list with the electricity requests of subsequent time intervals. However, the length of the profile may be shorter than the runtime if appliances have a periodic behavior. The profile is than used multiple times, after the last list element the first element is used again. For example, a refrigerator has a consumption profile with a length of approximately one hour, because it starts about once every hour, while it has a runtime of 24 hours per day.

We assume that appliances can only start once per simulation. When an appliance has to start twice in the planning period two appliances are defined.

\section{microCHP device}

The modeled microCHP device is a Whispergen ${ }^{\mathrm{TM}}$. We have chosen for this specific device since a Whispergen is available for testing. This is a Stirling engine based microCHP with only one generation level. Thus, the generation has two modes: on and off or $0 \mathrm{~W}$ and $1000 \mathrm{~W}$ [2]. When the microCHP device starts, it does not immediately generate the full $1000 \mathrm{~W}$. During the startup time (approx. $10 \mathrm{~min}$.) the generation increases from $0 \mathrm{~W}$ to $1000 \mathrm{~W}$. This is modeled as a linear increase. For stopping the same holds and we modeled this as a linear decrease from $1000 \mathrm{~W}$ to $0 \mathrm{~W}$. Once a microCHP device is started there is a minimum runtime before it can be switched off and when the microCHP device is stopped it cannot be started again within a minimum cool-down period.

The preferred status of the microCHP device at every time interval is determined by a controller. This controller can either be the standard thermostat or the balancing controller (see Section 2.3)

\section{Battery}

In first instance the electricity buffer is modeled as a battery, since this is the most common used buffer at the moment.

For simulating the battery the KiBaM (Kinetic Battery Model) [4] is used. This model keeps, next to the State of Charge (SoC), also track of maximum charge and discharge currents.

Within KiBaM, the battery is modeled as two charge containers, one representing the bound charge and the other the unbound charge (see Figure 3 ). The two containers are connected with a charge pipe with a limited diameter at the bottom. Charge can flow from the unbound container to the bound container and vice-versa via pipe $P_{1}$. The direction and speed of the flow depend on the fill-levels of both containers and is limited by the diameter of the pipe. Through

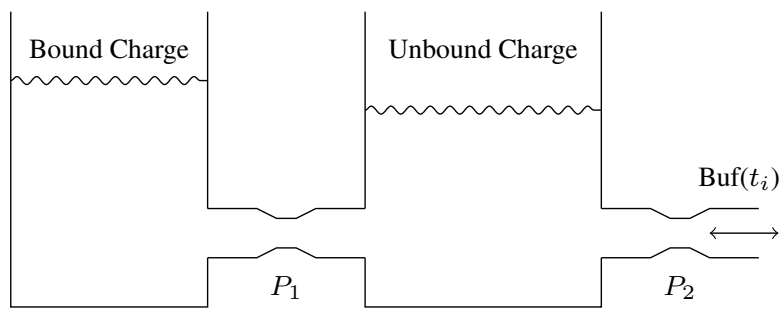

Figure 3. Schematic of the KiBaM battery model

another pipe $\left(P_{2}\right)$ in the unbound charge container, also with limited diameter, charge can flow in and out of the battery. It can be seen as if the containers were filled with water, this has the same behavior as the charge in the model.

Because of this setup the model implements a couple of characteristics of batteries that most models disregard, e.g. that the battery supplies more energy with lower discharge currents before it is empty and that when it is emptied with high currents it restores after a while. Since the clamp voltage depends on the unbound charge level a battery is empty when the clamp voltage drops below a certain level. Furthermore, the SoC and the maximum currents can be calculated by the (measured) clamp voltage.

\section{Grid}

During normal operation the import from and export to the grid are used for the balancing. When the generation of the microCHP device is lower than the demand of the appliances and the flow out of the battery, electricity is imported. When the generation is higher than the demand and flow into the battery, electricity is exported. So there is no balancing within the house, all mismatches are solved by the grid and the power plants have to take care of fluctuations.

Islanded operation implies that the house is disconnected from the grid so no import and export is possible. In that case the battery and balancing algorithm have to balance the demand and supply.

\subsection{Optimization decisions}

The aim of balancing algorithms for the Islanded House is, next to balancing the generation and consumption, decreasing the discomfort. Discomfort may be measured with help of the variables introduced in this section (see criteria and rating in Section 2.4). For the balancing and optimization the Islanded controller can decide when to start and stop the microCHP device and which appliances are supplied. 


\section{Appliances}

The consumption has two characteristics: demand and load. The demand is the total request of the appliances and the load the total accepted (supplied) demand and the export. Because of limitations on the amount and flexibility of available supply, the load will not always be equal to the demand. Therefore, in each time slice the controller has to decide which appliances are supplied. The demand of an appliance is in the previous section defined by the preferred start time, runtime and the profile. However, the control algorithms can decide to start appliances later (load shifting) or switch the appliance off during its runtime (preemption).

Since not all appliances allow preemption, the model also has to support non-preemtable appliances. Furthermore, some appliances are more important than others. To decide which appliances to supply, priorities are used. The goal is to find the best combination of appliances (considering the objective) taking into account that no high priority appliance is switched off for (multiple) lower priority appliances. However, non-preemption overrules priority.

\section{MicroCHP device}

During normal operation the standard heat controller decides whether to start the microCHP based on the heat demand and the status of the heat-store: the device is heat driven. To optimize the electricity streams switching on and off the microCHP device is electricity driven. However, the heat supply is the main purpose of a microCHP and gives limitations on the decision freedom: the heat must be produced before or at the moment it is consumed and the device cannot be switched on when the heatstore is full. Hence, during Islanded operation the goal is to decrease discomfort and energy efficiency comes second. Therefore, we assume that we can dump the heat so there are no limitations on the runtime. Furthermore, the minimum runtime and cooldown period give limitations on switching on and off the unit.

\subsection{Objectives and Criteria}

The developed algorithms work in an online fashion, i.e. every time slice the situation is optimized for that particular timeslice without considering the future. Since in this way the objective is only used to optimize a timeslice without awareness of the total result, the quality of the overall solution may not be optimal. Especially if no prediction is used the optimal solution for a time slice can have a negative influence on later time slices. As a consequence, optimization of time slices can have an unexpected influence on the total scheme.

The resulting schedules of the complete planning horizon are rated by criteria. These criteria rate the overall results and not individual time slices.

\section{Objective}

The Islanded house controller decides when to start/stop the microCHP device and which appliances are supplied. The objective in Islanded Operation focuses on which appliances are switched on and off, where the decision whether to start or stop the microCHP device is based on the system state.

For the decision which appliances are supplied in a time interval three different objectives are considered and simulated: maximize supply, maximize the number of appliances and minimize switching off appliances during runtime.

\section{Criteria}

There are two criteria considered for Islanded Operation, Quality of Comfort (QoC) and Quality of Supply (QoS). Quality of Comfort is a measure for the discomfort of the user. Quality of Supply is a measure for the energy efficiency on one hand and the wearing of the microCHP device and battery on the other hand. The two criteria itself are based on multiple parameters:

- Quality of Comfort

- Percentage of electricity demand supplied

- Shifted load (kWh)

- Number of times an appliance is switched off during runtime

- Quality of Supply

- Electricity loss (generation + SoC decrease - supply) (kWh)

- Number of times the microCHP device is started

- Supply by battery (kWh)

\subsection{Algorithm}

The algorithm has to control the microCHP device and decide which appliances are supplied. The basic inputs are the electricity buffer status, the microCHP device status, the electricity demand and priority/preemption information. Controlling the microCHP device and switching on/off appliances are two independent decisions since effects of switching on/off the microCHP device are only noticeable in the next time slice.

The first method for deciding to switch the microCHP device on or off bases the decision on whether the microCHP device can get rid of the generated electricity. The generator is switched on when the demand plus maximum charge current is higher than the generation level, otherwise it is switched off. The second method bases the decision 
on the maximum discharge current. When it drops below a certain level the machine is switched on, when it raises over a certain level the unit is switched off.

The exact trigger points (the value when to switch on/off) have a severe impact on the number of starts and the loss. These trigger points are determined by multiple simulations.

To decide which appliances are supplied, first the maximum available supply is determined by the generation level and maximum discharge. Next the best combination of appliances, based on the objective, is calculated.

\section{Results}

\subsection{Simulations}

We designed a simulator, based on the described model, to study the results of algorithms and parameters. In first instance the simulator uses six minute time slices. The length of the time slices is a tradeoff between accuracy and data usage/simulation time. According to [9] a five minute time slice is the best settlement, we used six minutes because it is $\frac{1}{10}$ of an hour.

The electricity usage profile is built up by the profiles of frequently used appliances. First, the profiles of frequently used appliances is deducted. Next realistic start- and runtimes are assigned in such a way that the total profile is realistic. The total profile is verified with the measured profiles in [9].

The simulations cover 24 hours, starting at 12 am with a fully charged battery. The aim of the simulations is to study the influence of different parameters on the overall performance of the system: switch on/off microCHP device decision method, battery capacity, preemption, priority and the objective for deciding which appliances are supplied. The reference case simulates Islanded operation for one day with a total demand of $12.5 \mathrm{kWh}$. The battery capacity is $80 \mathrm{Ah}$, all appliances are preemptable and all appliances have the same priority. Subsequently, the parameters are changed one by one in ten simulations:

2. on/off decision Base the on/off switching decision on the demand and maximum charge current.

3. Do not switch off Do not switch off the microCHP device.

4. Double battery A battery capacity of $160 \mathrm{Ah}$.

5. Half battery A battery capacity of $40 \mathrm{Ah}$.

6. Preemption 1 A non-preemptable washing machine and tumble dryer.

7. Preemption 2 A non-preemptable washing machine and computer appliances (computer, modem, etc.).
8. Priority 1 Higher priority for computer appliances and lighting.

9. Priority 2 Also higher priority for higher demand appliances (e.g. washing machine).

10. 2nd Objective Using the "maximizing number of appliances" objective.

11. 3th Objective Using the "minimizing switched off appliances" objective.

Based on the results of these simulations, a combination of parameters is chosen to create a best setting. This is a combination of simulation four and ten: Double battery capacity and the "maximizing number of appliances" objective.

\section{$3.2 \quad$ Results}

The results of the simulations are summarized in Table 1.

The alternative on/off switching decision for the microCHP device (case 2, 3) are both not superior to the reference case. Although the amount of shifted loads is lower in both cases, in case 2 the number of times the microCHP device is switched on is much higher. In case 3 the microCHP device is only switched on once and the number of appliances preempted lower, but the loss is much higher.

The battery capacity is an influencial parameter. More battery capacity decreases the amount of shifted load and the number of starts whereas the amount of battery discharge power does not increase significantly. Due to more capacity the battery can be charged and discharged for longer periods, so the microCHP device makes longer runs. More capacity also results in higher charge and discharge currents through which higher peaks can be supplied.

Non-preemption has a negative influence on the schedule, especially if these appliances have high demands. On the other hand, the amount of shifted electricity decreases because if for the high demand appliances preemption is allowed, they are more often shifted. It has to be noticed that non-preemptable appliances can lead to supply shortage; in the simulated scenarios such situations occurred.

The influence of priority depends on the way the priorities are assigned. On the one hand an optimal combination of appliances can violate the priority constraint but on the other hand a combination based on priority might be a better combination for future states. Therefore, it cannot be predicted whether priorities will have a positive or negative influence on the schedule. Nevertheless, priorities are required to define the most important (critical) appliances.

The objective for deciding which appliances are switched on/off is an important parameter for the schedule. The alternative objectives give overall better results. The second objective (maximizing the number of appliances) is 


\begin{tabular}{lccccccc}
\hline & \multicolumn{3}{c}{ Quality of Comfort } & & \multicolumn{3}{c}{ Quality of Supply } \\
\cline { 2 - 3 } & Supplied (\%) & Shifted (kWh) & $\begin{array}{c}\text { \# of } \\
\text { preemptions }\end{array}$ & & Loss (kWh) & $\begin{array}{c}\text { \# starts } \\
\text { microCHP }\end{array}$ & $\begin{array}{c}\text { Supply by } \\
\text { battery (kWh) }\end{array}$ \\
\hline 1) Reference & 99.3 & 12.2 & 92 & & 0.9 & 8 & 5.9 \\
2) on/off decision & 99.2 & 10.4 & 95 & & 2.8 & 12 & 5.3 \\
3) Do not switch off & 99.8 & 4.4 & 45 & & 10.9 & 1 & 3.6 \\
4) Double battery & 99.2 & 7.1 & 72 & & 0.4 & 4 & 6.2 \\
5) Half battery & 96.6 & 18.8 & 136 & & 2.6 & 13 & 4.9 \\
6) Preemption 1 & 97.7 & 8.8 & 94 & & 0.3 & 9 & 5.0 \\
7) Preemption 2 & 99.9 & 12.8 & 55 & & 0.6 & 7 & 5.7 \\
8) Priority 1 & 99.9 & 12.3 & 41 & & 0.8 & 7 & 5.9 \\
9) Priority 2 & 99.9 & 13.0 & 47 & & 0.8 & 7 & 5.9 \\
10) 2nd Objective & 100 & 11.5 & 26 & & 0.8 & 7 & 6.1 \\
11) 3th Objective & 100 & 14.2 & 24 & & 0.8 & 7 & 6.1 \\
12) 4) + 10) & 100 & 7.2 & 25 & & 0.4 & 4 & 6.3 \\
\hline
\end{tabular}

Table 1. Simulated criteria result for three changed parameters (see Section 3.1 for a description)

even better than the third (minimizing switching off appliances) because of the higher amount of shifted load with the third objective. Both objectives have simular results because maximizing the number of appliances leads to switching off only the high-demand appliances.

The last simulation shows that with a combination of a $2 \mathrm{kWh}$ battery and the second objective all demand can be supplied. However, there is still some discomfort because of the shifted load and preempted appliances. The loss is only three percent and the microCHP device is switched on only 4 times.

The results of the simulations have shown that, considering only the basic inputs, an undesirable usage and even a shortage of supply due to non-preemtable low priority appliances may occur. It also leads to non-optimal scheduling of the microCHP device, e.g. switching it off just before a peak. This can be improved by using predictions of the electricity demand. Two levels of prediction are possible: appliance level or total demand. Appliance level prediction uses the expected appliance usage profile to decide if an appliance can be supplied without leading to supply shortage and to improve the microCHP runtime schedule. Based on the expected total demand a rough profile is outlined with an amount of freedom to admit difference between expected and realized profile.

To summarize, simulations of the first versions of the algorithms show that Islanded operation with a microCHP device is possible without a lot of discomfort whereas adding prediction might lead to significant better results. However, the heat loss/dumping is not taken into account in the simulations.

\section{Acknowledgments}

This research is conducted within the Islanded House project supported by E.ON UK.

\section{References}

[1] http://www.plugwise.com.

[2] http://www.whispergen.com.

[3] A. de Jong, E.-J. Bakker, J. Dam, and H. van Wolferen. Technisch energie- en CO2-besparingspotentieel in Nederland (2010-2030). Platform Nieuw Gas, page 45, Juli 2006.

[4] M. J.F. and M. J.G. Lead acid battery storage model for hybrid energy systems. Solar Energy, 50(5):399-405, 1993.

[5] A. Molderink, V. Bakker, J. Hurink, and G. Smit. Islanded house operation using a micro chp. In 18th Annual Workshop on Circuits, pages 324-330. STW, Nov 2008.

[6] A. Peacock and M. Newborough. Controlling micro-chp systems to modulate electrical load profiles. Energy, 32(7):10931103, July 2007.

[7] E. Santi, D. Franzoni, A. Monti, D. Patterson, F. Ponci, and N. Barry. A fuel cell based domestic uninterruptible power supply. In IEEE Applied Power Electronics Conference and Exposition, page 605. IEEE, 2002.

[8] United States Department of Energy. The micro-CHP technologies roadmap. Results of the Micro-CHP Technologies Roadmap Workshop, December 2003.

[9] A. Wright and S. Firth. The nature of domestic electricityloads and effects of time averaging on statistics and onsite generation calculations. Applied Energy, 84(4):389-403, April 2007. 